\title{
Water maser detections in southern candidate post-AGB stars and planetary nebulae
}

\author{
O. Suárez ${ }^{1,2}$, J. F. Gómez ${ }^{2}$, L. F. Miranda ${ }^{2}$, J. M. Torrelles ${ }^{3}$, Y. Gómez ${ }^{4}$, G. Anglada ${ }^{2}$, and O. Morata ${ }^{5,6,7}$ \\ 1 UMR 6525 H.Fizeau, Université de Nice Sophia Antipolis, CNRS, OCA. Parc Valrose, 06108 Nice Cedex 2, France \\ e-mail: olga.suarez@unice.fr \\ 2 Instituto de Astrofísica de Andalucía, CSIC, Apartado 3004, 18080 Granada, Spain \\ 3 Instituto de Ciencias de Espacio (CSIC)-IEEC, Facultat de Física, Planta 7a, Universitat de Barcelona, Martí i Franquès 1, \\ 08028 Barcelona, Spain \\ ${ }^{4}$ Centro de Radioastronomía y Astrofísica, UNAM, Campus Morelia, Apdo. Postal 3-72, Morelia, Michoacán 58089, Mexico \\ 5 Department of Earth Sciences, National Taiwan Normal University, 88 Sec. 4, Ting Chou Rd., Taipei 116, Taiwan \\ 6 Academia Sinica Institute of Astronomy and Astrophysics, PO Box 23-141, Taipei 10617, Taiwan \\ 7 Departament d'Astronomía i Meteorología, Universitat de Barcelona, Martí i Franquès 1, 08028 Barcelona, Spain
}

Received 3 February 2009 / Accepted 8 June 2009

\section{ABSTRACT}

\begin{abstract}
Aims. We study the incidence and characteristics of water masers in the envelopes of stars in the post-AGB and PN evolutionary stages.

Methods. We used the 64-m antenna in Parkes (Australia) to search for water maser emission at $22 \mathrm{GHz}$, towards a sample of 74 sources with IRAS colours characteristic of post-AGB stars and PNe, at declination $<-32^{\circ}$. In our sample, $39 \%$ of the sources are PNe or PNe candidates, and 50\% are post-AGB stars or post-AGB candidates.

Results. We detected four new water masers, all of them in optically obscured sources: three are in PNe candidates (IRAS 12405-6219, IRAS 15103-5754, and IRAS 16333-4807) and one is in a post-AGB candidate (IRAS 13500-6106). The PN candidate IRAS 15103-5754 has water fountain characteristics and could be the first PN of this class found.

Conclusions. We confirm the trend suggested in Paper I that water maser emission during the post-AGB phase is more likely to be present in obscured sources with massive envelopes than in objects with optical counterpart. We propose an evolutionary scenario for water masers in the post-AGB and PNe stages, in which "water fountain" masers could develop during the post-AGB and early PN stages. Later PNe would exhibit lower velocity maser emission, both along jets and close to the central objects, with only the central masers remaining in more evolved PNe.
\end{abstract}

Key words. masers - surveys - stars: AGB and post-AGB - stars: mass-loss - planetary nebulae: general

\section{Introduction}

The study of $\mathrm{H}_{2} \mathrm{O}$ masers in evolved stars has proven to be a powerful tool to understand the evolution and shaping of sources between the asymptotic giant branch (AGB) and the planetary nebula phase $(\mathrm{PN})$. About $80 \%$ of the stars (those with $\left.M \leq 8 M_{\odot}\right)$ undergo these evolutionary stages, where a dramatic change in their morphology occurs. The stars in the AGB stage have a high mass-loss rate, which can reach $\simeq 10^{-4} M_{\odot} \mathrm{yr}^{-1}$. This mass-loss is usually spherical, although the first manifestations of asymmetry are already observed in some of them (see for example Leão et al. 2006; Josselin et al. 2000). A large fraction of PNe show, however, axial symmetries with bipolar or multipolar morphologies and, in many cases, jets (Sahai et al. 2007).

Water maser emission is frequently present in the AGB stage, where the spectra exhibit double-peak emission in some cases (e.g., Engels et al. 1986), similar to those of $\mathrm{OH}$ masers in this type of sources, which are characteristic of a spherical envelope expanding at velocities $\sim 10-30 \mathrm{~km} \mathrm{~s}^{-1}$ (Reid et al. 1977; Bowers et al. 1989). In AGB stars, we may also find $\mathrm{SiO}$ maser emission, and the three species are distributed in such a way that $\mathrm{SiO}$ masers are located close to the central star $(\sim 10 \mathrm{AU}), \mathrm{H}_{2} \mathrm{O}$ are located at the inner part of the envelope (between 10 and
$100 \mathrm{AU}$ ), and $\mathrm{OH}$ masers at distances $>10^{3} \mathrm{AU}$ from the central star (Reid \& Moran 1981).

In the classical view of maser emission being pumped by spherical AGB winds, $\mathrm{SiO}$ masers are expected to survive only $\simeq 10 \mathrm{yr}$ after the cessation of the strong mass-loss, while $\mathrm{H}_{2} \mathrm{O}$ and $\mathrm{OH}$ masers are extinguished after $\simeq 100 \mathrm{yr}$ and $\simeq 1000 \mathrm{yr}$, respectively (Lewis 1989; Gómez et al. 1990). These timescales imply that in the post-AGB stage we can still find some objects that harbour water masers with the same characteristics as those found in the AGB stage (see for example Engels 2002). On the other hand, water maser emission is unexpected in PNe.

There are cases, however, of evolved sources in which the observed water maser emission departs from the pattern expected from spherical mass-loss. In several post-AGB stars, called "water fountains", the $\mathrm{H}_{2} \mathrm{O}$ maser emission traces highly collimated bipolar jets with a velocity spread $\gtrsim 100 \mathrm{~km} \mathrm{~s}^{-1}$, and dynamical ages $\$ 100 \mathrm{yr}$ (Imai 2007), in what could represent the first manifestation of collimated mass-loss in evolved stars. We also now know of three confirmed cases of PNe harbouring water masers (Miranda et al. 2001; de Gregorio-Monsalvo et al. 2004; Gómez et al. 2008). These three " $\mathrm{H}_{2} \mathrm{O}-\mathrm{PNe}$ " all have a bipolar morphology, suggesting that the asymmetrical mass loss may be related to the presence of water masers in PNe. 
To study the possible relationships between the evolutionary stage of evolved objects and the presence of water masers, Suárez et al. (2007) (hereafter Paper I) carried out a single-dish survey for $\mathrm{H}_{2} \mathrm{O}$ maser emission towards the evolved sources in the catalogue of Suárez et al. (2006) (hereafter SGM) located north of declination $-32^{\circ}$. A total of 105 sources were searched for with the Robledo 70m antenna, with five detections, four of them reported in that paper for the first time. Three of these detections were found in obscured sources, of which one was a water fountain (confirmed by Suárez et al. 2008) and another a PN candidate. These results led us to suggest that water masers in the post-AGB and PN phases are more likely to exist in stars with more massive envelopes, and that PNe harbouring water masers are a special class of massive, rapidly evolving PNe. Water masers in these sources would be related to processes of non-spherical mass-loss, rather than being the remnant of water masers pumped by spherical winds in the AGB stage. In Paper I, it was also suggested that probably all $\mathrm{H}_{2} \mathrm{O}-\mathrm{PNe}$ had previously traversed a water fountain phase (see also Gómez et al. 2008). The discovery of objects exhibiting both water fountain and $\mathrm{PNe}$ characteristics would be important evidence of this evolutionary path.

In this paper, we present the continuation of work started in Paper I, with single-dish observations of sources in the SGM catalogue with declination $\leq-32^{\circ}$, using the Parkes antenna. This paper is structured as follows: In Sect. 2, we present the sample of observed sources. In Sect. 3, we describe the observations performed. In Sect. 4, we describe the results obtained, including a description of the detected objects. In Sect. 5, we discuss our results and finally, in Sect. 6, we give the main conclusions of this work.

\section{Description of the source sample}

The selection criteria are described in detail in Paper I and in SGM. Briefly, the SGM atlas consists of IRAS sources located in the region of the IRAS colour-colour diagram populated mainly by post-AGB stars (van der Veen et al. 1989). The sources in this region should have expanding envelopes characterised by a range of dust temperatures between $200 \mathrm{~K}$ and $80 \mathrm{~K}$, and radii between $0.01 \mathrm{pc}$ and $0.1 \mathrm{pc}$.

The evolved sources in the SGM catalogue are classified into five different groups: PN, post-AGB stars, transition sources (that are evolving between the post-AGB and PN phases), peculiar sources (evolved sources with peculiar characteristics), and objects with no optical counterpart (which could be evolved objects in any of the stages mentioned above that could not be classified with the optical spectroscopy presented in SGM).

For these objects with no optical counterpart found by SGM, we can only give a tentative classification as "post-AGB candidate" or "PN candidate". We consider them to be PN candidates when there is evidence of ionisation in the envelope, such as the presence of radio continuum emission. In many cases, these PN candidates are merely point-like sources in infrared images, with no further evidence of the presence of a nebula. When there is no indication of ionisation or nebulosity, we consider these obscured objects to be post-AGB candidates. Obviously, further infrared observations are required to ascertain the true nature of these objects without optical counterparts.

There are 106 evolved sources in the SGM catalogue located south of declination $-32^{\circ}$, which are the focus of this paper.
By the time the observations described in Sect. 3 were performed, we became aware of the paper by Deacon et al. (2007), who had carried out a search for water masers in postAGB candidates with $\mathrm{OH} 1662 \mathrm{MHz}$ maser emission. Their survey included 7 of the sources selected for our observations: IRAS 14341-6211, IRAS 17088-4221, IRAS 17164-3226, IRAS 17168-3736, IRAS 17245-3951, IRAS 17310-3432, and IRAS 17370-3357, and there was one water maser detection among them (IRAS 17088-4221). Therefore, these 7 sources were explicitly left out of our observations, although we include them in the discussion of this paper (see Sect. 5), whenever appropriate, because they fulfil the criteria used to select the sample.

\section{Observations}

We observed the $6_{16} \rightarrow 5_{23}$ transition of the water molecule (rest frequency $22235.080 \mathrm{MHz}$ ), using the $64 \mathrm{~m}$ antenna of the Parkes Observatory ${ }^{1}$, on 2007 February 08-12. At this frequency, the half-power beamwidth of the telescope is $\simeq 1 ! 3$. The $1.3 \mathrm{~cm}$ receiver of this antenna comprises a cryogenic High Mobility Electron Transistor. Both right and left circular polarisation were observed simultaneously. As a backend, we used the Multibeam Correlator, covering a bandwidth of $64 \mathrm{MHz}$ (i.e., velocity coverage $\simeq 862.9 \mathrm{~km} \mathrm{~s}^{-1}$ ) with 2048 spectral channels for each polarisation, thus yielding a spectral resolution of $31.25 \mathrm{kHz}\left(0.42 \mathrm{~km} \mathrm{~s}^{-1}\right)$. Since little information about the radial velocity of these sources is available, all observed spectra were centred on $V_{\mathrm{LSR}}=0 \mathrm{~km} \mathrm{~s}^{-1}$. The observations were taken in position-switching mode with a total integration time (on+off) of $30 \mathrm{~min}$. The rms pointing accuracy of the telescope was $\simeq 10^{\prime \prime}$.

Taking into account the detections found in Paper I, the priority of the observations was set (in decreasing order) to be: optically obscured sources, PNe, transition sources, and finally the post-AGB stars. A total of 74 objects out of the 106 target sources were observed. Table 1 provides the list of the observed objects. The target coordinates were taken from SGM, who list 2MASS positions when a near-IR counterpart of the IRAS sources was identified. When SGM does not provide these improved coordinates, we used those in the IRAS Point Source Catalog. The observed sources are divided into the following categories, according to their classification in SGM:

- 23 PNe with optical counterpart;

- 8 transition objects with optical counterpart;

- 15 post-AGB stars with optical counterpart;

- 28 sources without optical counterpart (6 PNe candidates, 22 post-AGB candidates).

These 74 sources plus the 7 objects observed by Deacon et al. (2007) (1 PN and 4 post-AGB stars with optical counterpart, and 2 post-AGB candidates without optical counterpart) comprised all objects without optical counterpart, as well as all PNe and transition objects with optical counterpart included in the selected sample of 106 sources from SGM.

\section{Results}

Our results are summarised in Table 1, where we list all the observed sources, and Table 2, where we provide the parameters

\footnotetext{
1 The Parkes telescope is part of the Australia Telescope, which is funded by the Commonwealth of Australia for operation as a National Facility managed by CSIRO.
} 
Table 1. Table of observed sources. The first day of observation 2007-FEB-08 corresponds to the Julian Date 2454139.5.

\begin{tabular}{|c|c|c|c|c|c|c|}
\hline IRAS name & $\overline{\text { RA (J2000) }}$ & $\overline{\text { Dec (J2000) }}$ & Observation date & rms (Jy) & $\begin{array}{c}\text { Classification } \\
\text { Suárez et al. (2006) }\end{array}$ & Notes \\
\hline $07027-7934$ & $06: 59: 26.4$ & $-79: 38: 47$ & 2007-FEB-09 & 0.22 & $\mathrm{PN}$ & \\
\hline 08046-3844 & 08:06:28.4 & $-38: 53: 24$ & 2007-FEB-10 & 0.19 & $\mathrm{PN}$ & \\
\hline 08242-3828 & $08: 26: 03.8$ & $-38: 38: 48$ & 2007-FEB-08 & 0.19 & No optical counterpart & 1 \\
\hline $08281-4850$ & 08:29:40.6 & $-49: 00: 04$ & 2007-FEB-12 & 0.21 & Post-AGB & \\
\hline 08351-4634 & $08: 36: 45.8$ & $-46: 44: 46$ & 2007-FEB-08 & 0.22 & No optical counterpart & \\
\hline 08355-4027 & $08: 37: 24.7$ & $-40: 38: 04$ & 2007-FEB-10 & 0.20 & $\mathrm{PN}$ & \\
\hline 08418-4843 & $08: 43: 29.5$ & $-48: 54: 47$ & 2007-FEB-10 & 0.17 & $\mathrm{PN}$ & \\
\hline 08574-5011 & 08:59:02.3 & $-50: 23: 40$ & 2007-FEB-10 & 0.17 & PN & \\
\hline $09362-5413$ & 09:37:51.8 & $-54: 27: 09$ & 2007-FEB-09 & 0.60 & $\mathrm{PN}$ & \\
\hline 09370-4826 & 09:38:53.3 & $-48: 40: 10$ & 2007-FEB-08 & 0.25 & No optical counterpart & \\
\hline 09425-6040 & 09:44:01.7 & $-60: 54: 26$ & 2007-FEB-12 & 0.23 & Post-AGB & \\
\hline $09500-5236$ & $09: 51: 49.2$ & $-52: 50: 53$ & 2007-FEB-08 & 0.22 & No optical counterpart & \\
\hline $09517-5438$ & $09: 53: 27.1$ & $-54: 52: 40$ & 2007-FEB-09 & 0.20 & $\mathrm{PN}$ & \\
\hline $10029-5553$ & 10:04:40.1 & $-56: 08: 37$ & 2007-FEB-09 & 0.21 & $\mathrm{PN}$ & \\
\hline $10115-5640$ & $10: 13: 19.7$ & $-56: 55: 32$ & 2007-FEB-09 & 0.22 & $\mathrm{PN}$ & \\
\hline $10178-5958$ & $10: 19: 32.5$ & $-60: 13: 29$ & 2007-FEB-10 & 0.17 & Transition & \\
\hline $10197-5750$ & $10: 21: 33.9$ & $-58: 05: 48$ & 2007-FEB-11 & 0.23 & Transition & \\
\hline $10215-5916$ & $10: 23: 19.5$ & $-59: 32: 05$ & 2007-FEB-11 & 0.25 & Transition & \\
\hline $10256-5628$ & $10: 27: 35.2$ & $-56: 44: 20$ & 2007-FEB-11 & 0.19 & Post-AGB & \\
\hline $11201-6545$ & $11: 22: 18.9$ & $-66: 01: 51$ & 2007-FEB-11 & 0.20 & Post-AGB & \\
\hline $11339-6004$ & $11: 36: 20.7$ & $-60: 20: 53$ & 2007-FEB-08 & 0.18 & No optical counterpart & \\
\hline $11353-6037$ & $11: 37: 42.9$ & $-60: 53: 51$ & 2007-FEB-11 & 0.22 & Transition & \\
\hline $11381-6401$ & $11: 40: 32.0$ & $-64: 18: 35$ & 2007-FEB-08 & 0.17 & No optical counterpart & \\
\hline $11387-6113$ & $11: 41: 08.7$ & $-61: 30: 17$ & 2007-FEB-11 & 0.22 & Post-AGB & \\
\hline $11531-6111$ & $11: 55: 38.0$ & $-61: 28: 17$ & 2007-FEB-11 & 0.22 & Transition & \\
\hline $12145-5834$ & 12:17:16.1 & $-58: 51: 30$ & 2007-FEB-12 & 0.24 & Post-AGB & \\
\hline $12262-6417$ & $12: 29: 04.2$ & $-64: 33: 37$ & 2007-FEB-08 & 0.18 & No optical counterpart & \\
\hline $12302-6317$ & $12: 33: 07.0$ & $-63: 33: 43$ & 2007-FEB-12 & 0.23 & Post-AGB & \\
\hline $12309-5928$ & $12: 33: 44.6$ & $-59: 45: 19$ & 2007-FEB-08 & 0.18 & No optical counterpart & \\
\hline $12316-6401$ & $12: 34: 36.0$ & $-64: 18: 17$ & 2007-FEB-09 & 0.23 & $\mathrm{PN}$ & \\
\hline $12405-6219$ & $12: 43: 31.5$ & $-62: 36: 14$ & 2007-FEB-08 & 0.12 & No optical counterpart & 2 \\
\hline $13203-5917$ & $13: 23: 32.2$ & $-59: 32: 50$ & 2007-FEB-12 & 0.24 & Post-AGB & \\
\hline $13293-6000$ & $13: 32: 39.2$ & $-60: 15: 39$ & 2007-FEB-08 & 0.18 & No optical counterpart & \\
\hline $13356-6249$ & $13: 39: 05.8$ & $-63: 04: 44$ & 2007-FEB-08 & 0.20 & No optical counterpart & \\
\hline $13416-6243$ & $13: 45: 07.3$ & $-62: 58: 17$ & 2007-FEB-12 & 0.24 & Post-AGB & \\
\hline $13427-6531$ & $13: 46: 25.7$ & $-65: 46: 24$ & 2007-FEB-08 & 0.20 & No optical counterpart & \\
\hline \multirow[t]{2}{*}{$13500-6106$} & $13: 53: 34.5$ & $-61: 20: 52$ & 2007-FEB-08 & 0.15 & No optical counterpart & \\
\hline & & & 2007-FEB-09 & 0.23 & & \\
\hline $13529-5934$ & $13: 56: 24.6$ & $-59: 48: 57$ & 2007-FEB-08 & 0.22 & No optical counterpart & \\
\hline $14079-6402$ & $14: 11: 46.3$ & $-64: 16: 24$ & 2007-FEB-09 & 0.22 & $\mathrm{PN}$ & \\
\hline \multirow[t]{2}{*}{$14122-5947$} & $14: 15: 53.3$ & $-60: 01: 38$ & 2007-FEB-10 & 0.13 & $\mathrm{PN}$ & \\
\hline & & & 2007-FEB-11 & 0.22 & & \\
\hline $14177-5824$ & $14: 21: 19.9$ & $-58: 38: 22$ & 2007-FEB-10 & 0.19 & $\mathrm{PN}$ & \\
\hline $14345-5858$ & $14: 38: 20.0$ & $-59: 11: 46$ & 2007-FEB-09 & 0.22 & PN & \\
\hline $14482-5725$ & $14: 51: 57.3$ & $-57: 38: 19$ & 2007-FEB-11 & 0.19 & Post-AGB & \\
\hline $14488-5405$ & $14: 52: 28.7$ & $-54: 17: 43$ & 2007-FEB-11 & 0.20 & Post-AGB & \\
\hline $15039-4806$ & $15: 07: 27.4$ & $-48: 17: 54$ & 2007-FEB-11 & 0.20 & Post-AGB & \\
\hline $15066-5532$ & $15: 10: 26.0$ & $-55: 44: 13$ & 2007-FEB-11 & 0.20 & Transition & \\
\hline $15093-5732$ & $15: 13: 12.4$ & $-57: 43: 40$ & 2007-FEB-08 & 0.21 & No optical counterpart & \\
\hline \multirow[t]{2}{*}{$15103-5754$} & $15: 14: 18.5$ & $-58: 05: 20$ & 2007-FEB-09 & 0.24 & No optical counterpart & 2 \\
\hline & & & 2007-FEB-12 & 0.6 & & \\
\hline $15144-5812$ & $15: 18: 21.9$ & $-58: 23: 12$ & 2007-FEB-09 & 0.22 & No optical counterpart & \\
\hline $15154-5258$ & $15: 19: 08.2$ & $-53: 09: 47$ & 2007-FEB-10 & 0.17 & PN & \\
\hline $15210-6554$ & $15: 25: 31.7$ & $-66: 05: 20$ & 2007-FEB-11 & 0.19 & Post-AGB & \\
\hline $15534-5422$ & $15: 57: 21.1$ & $-54: 30: 46$ & 2007-FEB-09 & 0.21 & No optical counterpart & \\
\hline $15559-5546$ & $15: 59: 57.4$ & $-55: 55: 34$ & 2007-FEB-10 & 0.18 & $\mathrm{PN}$ & \\
\hline $15579-5445$ & $16: 01: 50.8$ & $-54: 53: 40$ & 2007-FEB-10 & 0.17 & $\mathrm{PN}$ & \\
\hline $16053-5528$ & $16: 09: 20.2$ & $-55: 36: 10$ & 2007-FEB-10 & 0.17 & PN & \\
\hline $16114-4504$ & $16: 15: 03.0$ & $-45: 11: 54$ & 2007-FEB-10 & 0.18 & PN & \\
\hline $16228-5014$ & $16: 26: 31.3$ & $-50: 21: 27$ & 2007-FEB-09 & 0.22 & No optical counterpart & \\
\hline $16333-4807$ & 16:37:06.1 & $-48: 13: 42$ & 2007-FEB-09 & 0.20 & No optical counterpart & 2 \\
\hline $16494-3930$ & $16: 52: 55.4$ & $-39: 34: 56$ & 2007-FEB-10 & 0.20 & Post-AGB & \\
\hline $16518-3425$ & $16: 55: 08.4$ & $-34: 30: 10$ & 2007-FEB-09 & 0.23 & No optical counterpart & \\
\hline
\end{tabular}


Table 1. continued.

\begin{tabular}{|c|c|c|c|c|c|c|}
\hline IRAS name & $\overline{\overline{\text { RA (J2000) }}}$ & 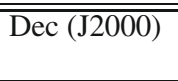 & Observation date & $\overline{\text { rms (Jy) }}$ & $\begin{array}{c}\text { Classification } \\
\text { Suárez et al. (2006) } \\
\end{array}$ & Notes \\
\hline $16529-4341$ & $16: 56: 34.0$ & $-43: 46: 15$ & 2007-FEB-10 & 0.18 & $\mathrm{PN}$ & \\
\hline $16584-3710$ & 17:01:52.1 & $-37: 14: 54$ & 2007-FEB-09 & 0.21 & No optical counterpart & \\
\hline $16594-4656$ & 17:03:11.2 & $-47: 00: 21$ & 2007-FEB-10 & 0.19 & Transition & \\
\hline 17009-4154 & 17:04:29.6 & $-41: 58: 39$ & 2007-FEB-09 & 0.22 & No optical counterpart & \\
\hline $17010-3810$ & $17: 04: 27.3$ & $-38: 14: 42$ & 2007-FEB-09 & 0.22 & No optical counterpart & \\
\hline $17088-4227$ & $17: 12: 21.8$ & $-42: 30: 50$ & 2007-FEB-10 & 0.18 & $\mathrm{PN}$ & \\
\hline 17119-5926 & $17: 16: 21.1$ & $-59: 29: 23$ & 2007-FEB-10 & 0.17 & PN & \\
\hline $17130-4029$ & $17: 16: 29.0$ & $-40: 32: 31$ & 2007-FEB-09 & 0.20 & No optical counterpart & \\
\hline $17131-3330$ & $17: 16: 26.2$ & $-33: 33: 24$ & 2007-FEB-10 & 0.21 & $\mathrm{PN}$ & \\
\hline 17153-3814 & $17: 18: 44.7$ & $-38: 17: 21$ & 2007-FEB-09 & 0.21 & No optical counterpart & 3 \\
\hline $17234-4008$ & $17: 26: 56.1$ & $-40: 11: 04$ & 2007-FEB-09 & 0.22 & No optical counterpart & \\
\hline $17311-4924$ & $17: 35: 02.5$ & $-49: 26: 26$ & 2007-FEB-10 & 0.18 & Transition & \\
\hline 17418-3335 & $17: 45: 08.7$ & $-33: 36: 06$ & 2007-FEB-09 & 0.20 & No optical counterpart & 3 \\
\hline $17476-4446$ & $17: 51: 16.4$ & $-44: 47: 29$ & 2007-FEB-10 & 0.19 & Post-AGB & \\
\hline
\end{tabular}

${ }^{1} \mathrm{OH}$ maser emission detected by Te Lintel Hekkert et al. (1991). The spectrum does not exhibit the typical double-peaked profile of OH/IR stars, but contains multiple components. This suggests that the source is a post-AGB star; ${ }^{2}$ radio continuum emission detected by van de Steene $\&$ Pottasch (1993). Thus, we consider this source to be a "PN candidate"; ${ }^{3}$ radio continuum emission detected by Ratag et al. (1990). Thus, we consider this source to be a "PN candidate".

Table 2. Water maser detections.

\begin{tabular}{ccccc}
\hline \hline IRAS & $\begin{array}{c}V_{\text {peak }} \\
\left(\mathrm{km} \mathrm{s}^{-1}\right)\end{array}$ & $\begin{array}{c}S_{\text {peak }} \\
(\mathrm{Jy})\end{array}$ & $\begin{array}{c}\int S_{\nu} \mathrm{d} v \\
\left(\mathrm{Jy} \mathrm{km} \mathrm{s}^{-1}\right)\end{array}$ & Date \\
\hline $12405-6219$ & $-23.6 \pm 0.4$ & $0.94 \pm 0.24$ & $1.5 \pm 0.4$ & 2007 -FEB-08 \\
$13500-6106$ & $39.9 \pm 0.4$ & $0.8 \pm 0.3$ & $1.2 \pm 0.5$ & 2007 -FEB-08 \\
& $40.0 \pm 0.4$ & $1.6 \pm 0.5$ & $0.9 \pm 0.7$ & 2007 -FEB-09 \\
$15103-5754$ & $-35.8 \pm 0.4$ & $60.3 \pm 0.5$ & $179 \pm 3$ & $2007-F E B-09$ \\
& $-35.8 \pm 0.4$ & $36.7 \pm 1.2$ & $95 \pm 8$ & 2007 -FEB-12 \\
$16333-4807$ & $-42.2 \pm 0.4$ & $1.8 \pm 0.4$ & $3.5 \pm 0.7$ & $2007-F E B-09$ \\
\hline
\end{tabular}

of the detections obtained. Four water masers were found during these observations, all of them reported here for the first time: IRAS 12405-6219, IRAS 13500-6106, IRAS 15103-5754, and IRAS 16333-4807. Their spectra are shown in Figs. 1 and 2. All of them correspond to objects classified as "sources without optical counterpart" by SGM.

The sources IRAS 12405-6219, IRAS 15103-5754, and IRAS 16333-4807 were classified by van de Steene \& Pottasch (1993) as PN candidates because of the presence of radio continuum emission, while IRAS 13500-6106 was classified as a postAGB star by García-Lario et al. (1997), based on its position in the IRAS two-colour diagram and on its low IRAS variability index $(v=29)$.

The association of the discovered water masers towards IRAS 12405-6219, IRAS 15103-5754, and IRAS 16333-4807 with the radio continuum detected by van de Steene \& Pottasch (1993) should be carefully studied. The beam of our water maser observations is 1.3 , while that of the radio continuum observations is 4".5. An unambiguous identification of the water maser emission with the radio continuum sources would require higher angular resolution observations of the water masers to derive a more accurate position (see, e.g., Gómez et al. 2008).

In the following we describe in detail the four detected objects and comment on their nature. To identify possible candidates to the maser-exciting source, we also inspected MSX and 2MASS data.

\subsection{Detected sources}

\subsubsection{IRAS 12405-6219}

We detected water maser emission with a single spectral component at $V_{\mathrm{LSR}} \simeq-23.6 \mathrm{~km} \mathrm{~s}^{-1}$ and a flux density of $\sim 1 \mathrm{Jy}$ (Fig. 1 and Table 2). A source of continuum emission at $6 \mathrm{~cm}$ was detected by van de Steene \& Pottasch (1993) at $\mathrm{RA}(\mathrm{J} 2000)=12^{\mathrm{h}} 43^{\mathrm{m}} 32.04^{\mathrm{s}}, \operatorname{Dec}(\mathrm{J} 2000)=-62^{\circ} 36^{\prime} 13.9^{\prime \prime}$, with a flux density $S(6 \mathrm{~cm})=14 \mathrm{mJy}$. This radio source is located $6^{\prime \prime}$ from the IRAS nominal position.

There is only one MSX source in the beam of the water maser observations. This source (MSX6C G302.0213+00.2542) is located $\sim 6^{\prime \prime}$ from the reported radio continuum position, and is coincident with the 2MASS source J12433151-6236135. We can consider that the radio continuum is associated with the IR source within the errors. The water maser position is also probably associated with this source.

Although van de Steene \& Pottasch (1993) classify it as a possible PN, this source also fulfils the criteria used by Hughes \& MacLeod (1989) to select HII regions from the IRAS Point Source Catalogue $\left(\log S_{60} \mu \mathrm{m} / \log S_{25} \mu \mathrm{m} \geq 0.25\right.$ and $\left.\log S_{25 \mu \mathrm{m}} / \log S_{12 \mu \mathrm{m}} \geq 0.4\right)$. Therefore its classification as $\mathrm{PN}$ is doubtful.

\subsubsection{IRAS $13500-6106$}

Our water maser spectrum shows one component with a maximum flux density of $\sim 1.5 \mathrm{Jy}$ at $V_{\mathrm{LSR}} \simeq 40.0 \mathrm{~km} \mathrm{~s}^{-1}$ (see Fig. 1 and Table 2). This source was classified as a post-AGB star by García-Lario et al. (1997) based on its IR colours. The only MSX source in the beam of the water maser observations is MSX6C G310.3099+00.6306, which has no associated 2MASS source. No $\mathrm{OH}$ was detected in this source by Te Lintel Hekkert et al. (1991).

\subsubsection{IRAS $15103-5754$}

We detected water maser emission with a maximum flux density of $\sim 60$ Jy (Fig. 1 and Table 2). The spectrum shows a rich set of multiple components spanning $\simeq 80 \mathrm{~km} \mathrm{~s}^{-1}$, from $V_{\mathrm{LSR}} \simeq-70$ to $+10 \mathrm{~km} \mathrm{~s}^{-1}$. In Fig. 2, we show a close-up view of the spectrum, 

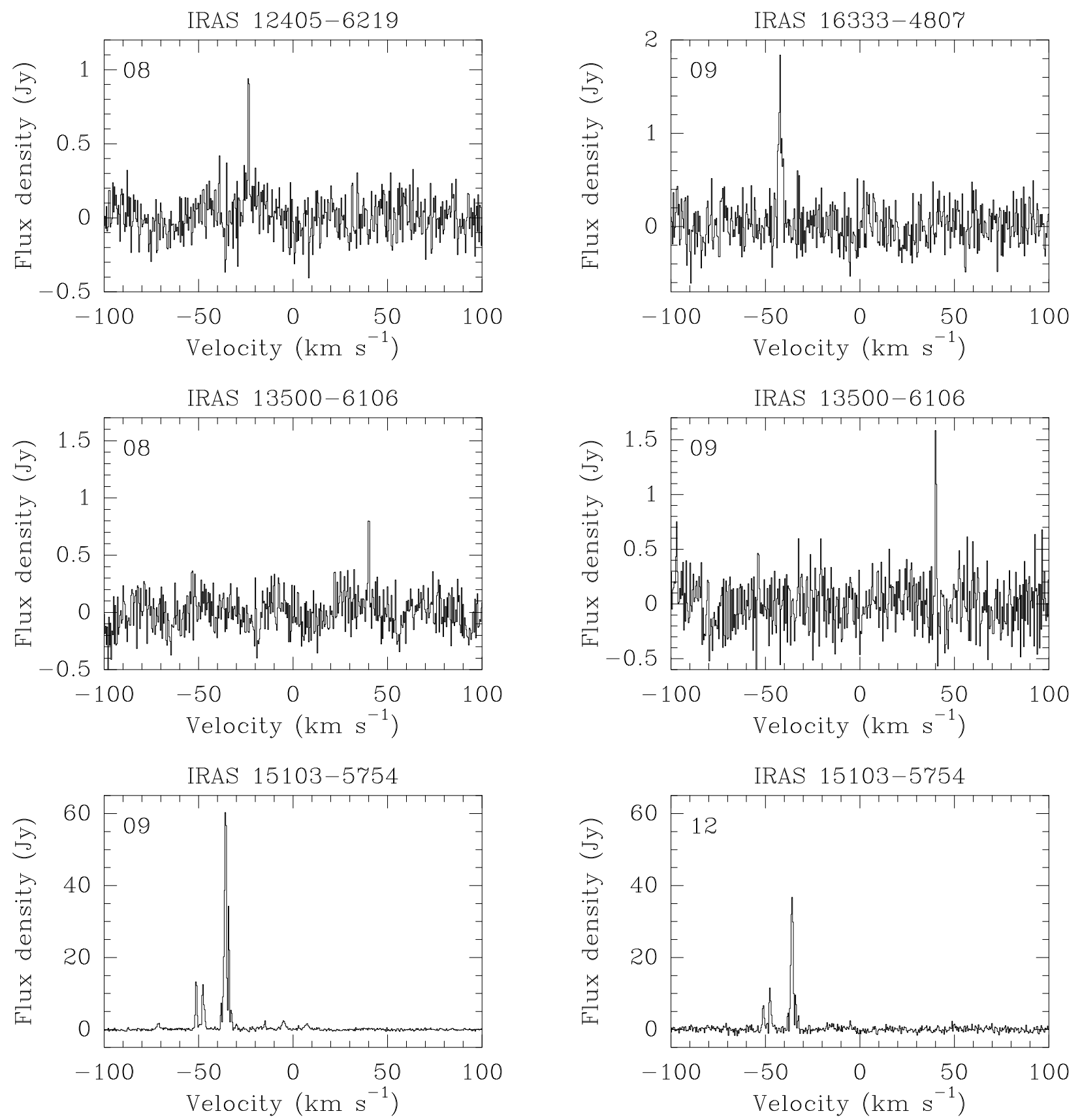

Fig. 1. Water maser spectra of the detected sources. The numbers shown at the upper left of the graph correspond to the day of observation in 2007 February.

where we can see the weakest components. The intensity of the components shows significant variability ( of up to a factor of $\simeq 3$ ) in the spectra taken on two different days.

The radio continuum source reported by van de Steene \& Pottasch (1993) is located at $\mathrm{RA}(\mathrm{J} 2000)=15^{\mathrm{h}} 14^{\mathrm{m}} 18.53^{\mathrm{s}}$, $\operatorname{Dec}(\mathrm{J} 2000)=-58^{\circ} 05^{\prime} 21.1^{\prime \prime}$, i.e., $15^{\prime \prime}$ from the IRAS nominal position, and has a flux density $S(6 \mathrm{~cm})=115 \mathrm{mJy}$. OH maser emission at $1612 \mathrm{MHz}$ was detected in this source (Te Lintel Hekkert \& Chapman 1996) at $V_{\mathrm{LSR}} \simeq-47 \mathrm{~km} \mathrm{~s}^{-1}$ with a flux density $\sim 0.3 \mathrm{Jy}$. The only MSX source in the beam of our observations is MSX6C G320.9062-00.2928, which is coincident with the 2MASS source J15141845-5805203 (to within 1'.'2). The MSX source is also coincident with the radio continuum position (within $\sim 1$ ". 4). These results suggest that IRAS 15103-5754 is likely to be a PN, associated with the aforementioned IR sources.

The high velocity range found in the water maser components makes this source a new candidate of the water fountain class. If its classification as a PN and its association with the water maser emission are indeed confirmed, this source would be the first PN with water fountain characteristics. The existence of "water fountain-PNe" was suggested by Gómez et al. (2008). They discussed the possibility of finding a PN that would contain the high-velocity water masers present during its previous postAGB-water fountain stage. These objects should be among the youngest and more massive PNe known, and they would be excellent targets for studying the "real-time" evolution of a PN, since the timescales of evolution of this type of sources would 


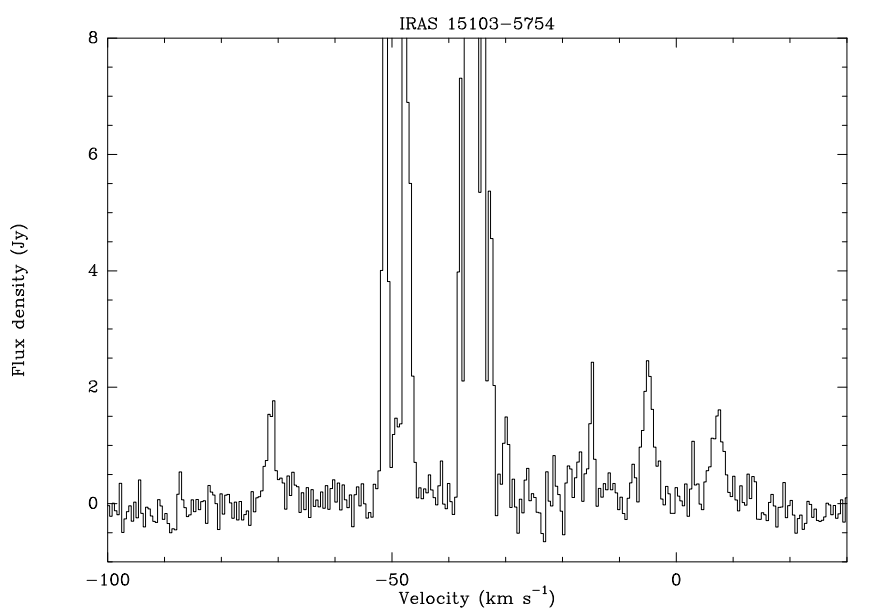

Fig. 2. Close up view of the water maser spectrum taken on 2007 February 9 toward the PN candidate IRAS 15103-5754.

be very short and can be studied by means of high angular resolution observations of the water masers.

\subsubsection{IRAS 16333-4807}

We detected water maser emission towards this source (see Fig. 1 and Table 2) with a maximum flux density of $\sim 1.9 \mathrm{Jy}$ at $V_{\mathrm{LSR}} \simeq 45 \mathrm{~km} \mathrm{~s}^{-1}$. The radio continuum source detected by van de Steene \& Pottasch (1993) is located at $\mathrm{RA}(\mathrm{J} 2000)=16^{\mathrm{h}} 37^{\mathrm{m}} 06.79^{\mathrm{s}}, \operatorname{Dec}(\mathrm{J} 2000)=-48^{\mathrm{o}} 13^{\prime} 42.6^{\prime \prime}$, displaced $\simeq 7^{\prime \prime}$ from the IRAS nominal position, with a flux density $S(6 \mathrm{~cm})=32.5 \mathrm{mJy}$.

The only MSX source in the beam of our observations is MSX6C G336.6445-00.6953, which is coincident with the source found by van de Steene \& Pottasch (1993) (to within $\sim 1{ }^{\prime \prime} 4$ ) and with the 2MASS source J16370659-4813429 (to within $\sim 1$ '. 5 ). The likely association of the radio continuum with IR sources makes this object a plausible PN candidate.

\subsection{Results from the two surveys for water masers in SGM sources}

The 179 sources observed in both campaigns (Robledo [Paper I] and Parkes [this paper]) are distributed in evolutionary classes, according to SGM, as follows:

- 76 post-AGB stars with optical counterpart (42\%);

- 21 transition sources (12\%);

- 35 PN with optical counterpart (20\%);

- 47 sources without optical counterpart (2 HII regions, 35 post-AGB candidates, $10 \mathrm{PN}$ candidates) (26\%).

And the detections have been found towards:

- 1 post-AGB star with optical counterpart: IRAS 07331+0021;

- 3 post-AGB candidates without optical counterpart, one of them a "water fountain": IRAS 16552-3050 (water fountain), IRAS 13500-6106, and IRAS 17580-3111;

- 1 confirmed PN with optical counterpart: IRAS 18061-2505;

- 3 PNe candidates without optical counterpart, one of them a possible "water fountain PN": IRAS 12405-6219, IRAS 15103-5754 (water fountain PN candidate), and IRAS 16333-4807;
- 1 AGB star without optical counterpart (OH/IR): IRAS 17443-2949.

We note that IRAS 17580-3111 and IRAS 17443-2949 were initially classified as obscured $\mathrm{PNe}$ with $\mathrm{OH}$ emission (OHPN) by Zijlstra et al. (1989), and as such were cited in Suárez et al. (2007), but Gómez et al. (2008) showed that the radio continuum reported by Ratag et al. (1990) was not associated with these sources, and García-Hernández et al. (2007) classified them as post-AGB and AGB stars, respectively. We adopt the classification of García-Hernández et al. (2007) in this discussion.

In summary, out of the nine detected sources, only one turned out to be a "classical" post-AGB star with an optical counterpart. The remaining possible post-AGB stars in which $\mathrm{H}_{2} \mathrm{O}$ emission was found do not have a counterpart in the visible domain. Since these stars could already be in the post-AGB stage while still having a thick envelope that prevents their detection in the optical, they may be relatively massive stars that have quickly evolved from the AGB. The new detections found in this campaign confirm the trend already found in Paper I, relating the presence of water masers in post-AGB stars or PN to optically obscured sources.

Apparently, the incidence of water maser emission during the post-AGB phase is higher in obscured objects, i.e., those with more massive envelopes. Of the 80 visible post-AGB stars observed, only 1 was found to have water maser emission $(\sim 1 \%)$, while we found this emission in 4 out of 35 post-AGB star candidates without optical counterparts $(\sim 11 \%)$. This suggests that the presence of massive envelopes favour the pumping of water masers during the post-AGB stage. Our water maser detections towards three obscured PN candidates might suggest a similar trend during the PN phase. The detection of dense molecular gas toward an $\mathrm{H}_{2} \mathrm{O}-\mathrm{PN}$ (Tafoya et al. 2007) also supports the existence of massive envelopes in these objects.

The two most interesting results of our two surveys are, on the one hand, the detection of several new possible $\mathrm{PNe}$ with water maser emission (three candidates found in this paper), and on the other hand, the possibility of having found the first "water fountain-PN". If the association of the water masers with the reported radio continuum emission is confirmed in the three candidates found in this paper (IRAS 12405-6219, IRAS 15103-5754, and IRAS 16333-4807), the number of PNe known to harbour water maser emission would increase from 3 to 6 . We expect these new $\mathrm{H}_{2} \mathrm{O}-\mathrm{PNe}$ to be bipolar, as is the case for the other three known to belong to this group. High resolution studies of these objects would be necessary to confirm this hypothesis. Furthermore, the confirmation of IRAS 15103-5754 as the first "water fountain-PN" would lead us to define a new class of objects susceptible to being one of the youngest and more massive PNe known.

\section{Discussion}

The original purpose of this work was to study the connection between the presence of water masers and the evolution of the stars in the post-AGB stage. This was the reason why the objects to be observed were selected from the SGM catalogue, since this catalogue contains the largest number of post-AGB stars with spectroscopic classification compiled to date. However, as mentioned in Paper I, we have to be careful in deriving conclusions from the $\mathrm{H}_{2} \mathrm{O}$ maser detection rate obtained in objects without optical counterpart, since the SGM catalogue is incomplete for optically obscured sources. However, the fact that only $26 \%$ of the total sample consists of obscured objects, but $78 \%$ of the 


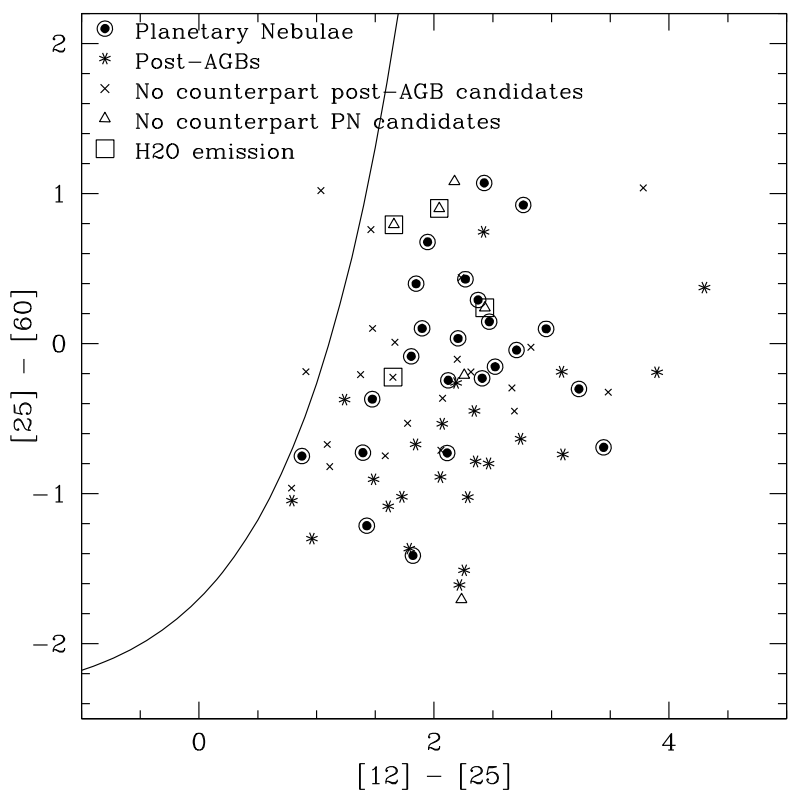

Fig. 3. IRAS two-colour diagram with the position of the observed and detected sources in the Parkes survey. The solid line is the curve modelled by Bedijn (1987) showing the position of the AGB stars.

detections have been found in these sources, strongly suggests that the presence of massive envelopes favours the pumping of water masers in the post-AGB or PN stages.

\subsection{Position of the detected sources in the IRAS and MSX colour-colour diagram}

To study the evolutionary status of the sources associated with water masers, we plotted them in both the IRAS [12]-[25] vs. [25]-[60] diagram and in the MSX [8]-[12] vs [15]-[21] diagram.

Figure 3 shows the position in the IRAS two-colour diagram of all the 74 sources observed in this paper. We use different symbols for the different types of sources and we also indicate the objects for which water masers have been found. In Fig. 4, we show the position in the IRAS two-colour diagram of the 9 sources with water masers detected in both surveys (Robledo and Parkes). We also include the post-AGB candidate IRAS 17088-4221, detected by Deacon et al. (2007) and, for completeness, we also include the position of the other two $\mathrm{H}_{2} \mathrm{O}$ PN, IRAS 17347-3139 (de Gregorio-Monsalvo et al. 2004) and PN K3-35 (Miranda et al. 2001). In this figure, we have used the same symbol for confirmed and candidate water-maser-emitting $\mathrm{PNe}$, and analogously for confirmed and candidate post-AGB stars.

A trend is apparent in Fig. 4, with confirmed and candidate $\mathrm{H}_{2} \mathrm{O}$-PNe having a larger value of [25]-[60] colour than watermaser-emitting post-AGB stars and candidates. However, a confirmation of this trend will require a more complete sample, especially for optically obscured evolved stars and, of course, a conclusive determination of the association of masers with the candidate objects, and the true nature of these.

In Fig. 5, we show the position in the [8]-[12] vs [15]-[21] MSX two-colour diagram of all the detections in both surveys that have been observed with the MSX satellite (all but IRAS $07331+0021$ and IRAS $16552-3050$ ), plus the other two $\mathrm{H}_{2} \mathrm{O}$ PNe and the post-AGB candidate IRAS 17088-4221. This diagram was divided into four quadrants (QI, QII, QIII, QIV)

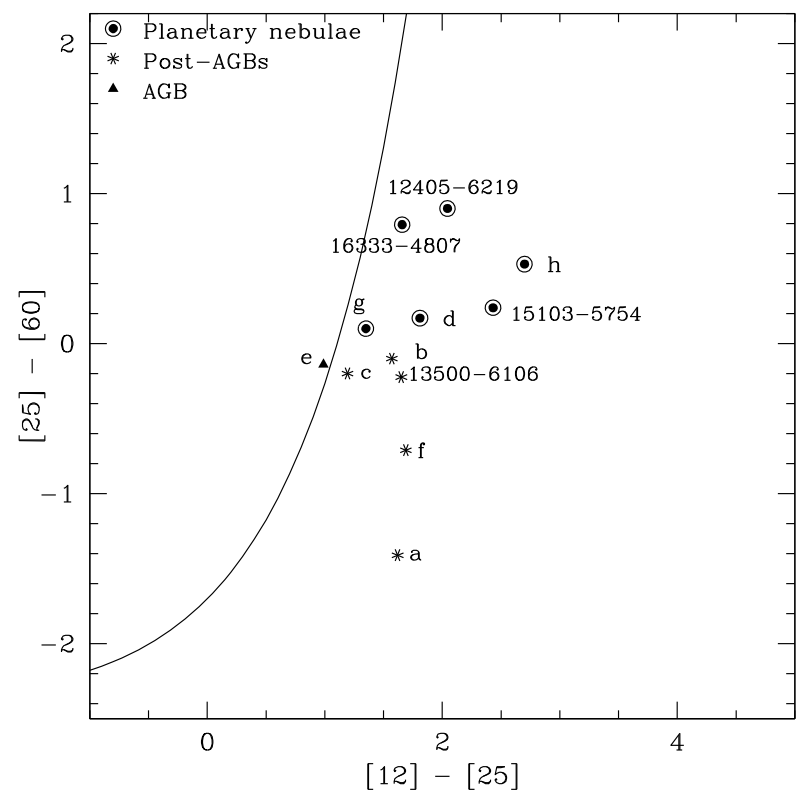

Fig. 4. IRAS-two colour diagram with the position of the detected sources in both campaigns (Robledo and Parkes detections). We have also included the position of the other two $\mathrm{H}_{2} \mathrm{O}$-PN known (IRAS 17347-3139, and K3-35), and of IRAS 17088-4221. The sources detected in the present campaign are shown with the IRAS names, while the rest of the sources are identified by labels that correspond to: a. IRAS 07331+0021, b. IRAS 16552-3050, c. IRAS 17088-4221, d. IRAS 17347-3139, e. IRAS 17443-2949, f. IRAS 17580-3111, g. IRAS 18061-2505 h. K3-35. The solid line is the curve modelled by Bedijn (1987) showing the position of the AGB stars. Note that in this figure, we are using the same symbols for confirmed and candidate sources in each stage.

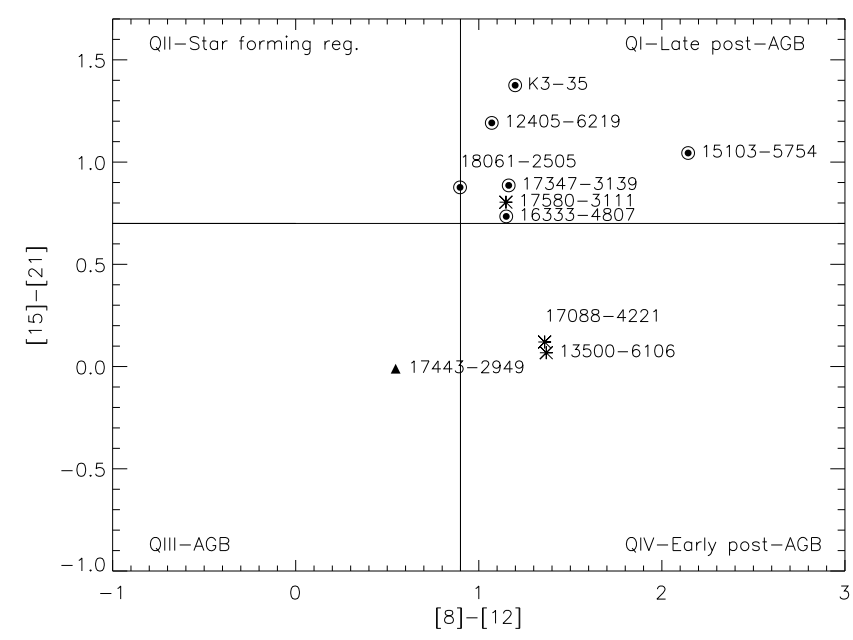

Fig. 5. MSX two-colour diagram with the position of the detected sources in both campaigns (Robledo and Parkes detections). We have also included the position of the other two $\mathrm{H}_{2} \mathrm{O}-\mathrm{PN}$ known (IRAS 17347-3139 and K3-35), and of IRAS 17088-4221. The symbols are the same as those defined in Fig. 4.

by Sevenster (2002) in her study of the position of OH-maseremitting AGB and post-AGB sources. The four quadrants correspond to the position of classical AGB stars (QIII, low left), early post-AGB stars (QIV, low right), more evolved objects (QI, up right), and star-forming regions (QII, up left). 
Although the defined quadrants provide only a tentative classification of the sources located in each of them (e.g., we can also find post-AGB stars and PNe in QII and QIII, see Suárez 2004), we note that IRAS 17443-2949 is the only source that occupies its place in QIII, the AGB quadrant, which supports its identification by García-Hernández et al. (2007) as an AGB star. All the $\mathrm{PNe}$ and $\mathrm{PNe}$ candidates are located in the region QI, occupied by evolved post-AGB and PNe, and the only objects located in the region QIV (early post-AGB stars) are the post-AGB candidates IRAS 13500-6106 and IRAS 17088-4221. Since there are no objects in QII, it is likely that none of our detections is a star-forming region, even if we maintain our doubts about the PN nature of IRAS 12405-6219.

\subsection{Evolutionary consequences of the presence of water masers in post-AGB stars and $P N e$}

As already stated in Lewis (1989) and Gómez et al. (1990), the conditions of $\mathrm{H}_{2} \mathrm{O}$ maser pumping in the AGB phase can continue during the post-AGB stage for at most $\sim 100 \mathrm{yr}$ in the case of spherical mass-loss. Therefore, this timescale indicates that most $\mathrm{H}_{2} \mathrm{O}$ masers found in post-AGB stars are probably originated during this phase.

As suggested both in Paper I and Gómez et al. (2008), the presence of asymmetrical mass-loss in the post-AGB stage can favour the necessary conditions to pump water masers in this phase and in that of PN. This is independent of the previous presence or not of these masers during the AGB phase, as in the case of the water fountains, such as IRAS 16552-3050 (Suárez et al. 2008), first detected in Paper I. If the three new obscured PNe candidates found to harbour water masers in this paper turn out to have bipolar morphologies, as for the other three $\mathrm{H}_{2} \mathrm{O}-\mathrm{PNe}$ known, it will help us to support the suggestion made both in Paper I and Gómez et al. (2008) relating the presence of water masers at the $\mathrm{PN}$ stage (the $\mathrm{H}_{2} \mathrm{O}-\mathrm{PNe}$ ) to the phenomenon of water fountains. Moreover, the discovery in this survey of the PN candidate IRAS 15103-5754 with "water fountain" characteristics provides an excellent evidence of the adequacy of the evolutionary model proposed, since its existence was predicted in Gómez et al. (2008). This source, if definitively confirmed as a "water fountain-PN", would be the missing link between the "water fountains" and the $\mathrm{H}_{2} \mathrm{O}-\mathrm{PNe}$.

A proposed scenario for the maser emission in the "water fountains" (Imai et al. 2007) suggests that after the water fountain is formed, the bipolar jets that harbour the water masers will reach the region of the former AGB envelope where the density would not be high enough to maintain the pumping of the masers. However, as discussed in Gómez et al. (2008), the regions close to the equator of the star, could still fulfil the conditions required to pump the water masers, even when the central star has reached a high enough temperature to become a PN. If the star is massive enough to evolve rapidly through these stages, we could still see the water fountain when it has reached the PN stage. This could be the case for IRAS 15103-5754, if high resolution observations confirm that the high velocity maser components originate in bipolar jets.

K3-35 should correspond to the next evolutionary stage, in which the jets would have been decelerated before the quenching of the water masers in the lobes of the PN. The remainder of the $\mathrm{H}_{2} \mathrm{O}-\mathrm{PNe}$ detected until now would correspond to the final stage when the masers are only pumped in dense areas close to the central stars.

Following the discussion in Gómez et al. (2008), we suggest a tentative scenario and its corresponding characteristics for the evolution of the water masers in the post-AGB and PN stages:

- Post-AGB stars with water maser emission that does not trace high velocity jets (e.g., IRAS $07331+0021$ ). In a few cases, this maser emission might be the remnant of the one pumped during the AGB phase. In others, however, the emission may have been produced during the post-AGB phase itself, but the source characteristics do not favour maser amplification in jets, or do not permit the jet formation.

- Post-AGB stars that show water fountain characteristics (e.g., IRAS 16552-3050; 11 sources known, 9 of them obscured in the optical).

- Young bipolar PN with water fountain characteristics (e.g., IRAS 15103-5754; this is the only candidate known up to now and it is obscured in the optical range).

- Young bipolar PN showing water masers at the tips of the bipolar lobes and at close to the central star (e.g., PN K3-35; this is the only source that has shown these characteristics, in one epoch).

- Young bipolar PN with water masers only close to the central star (e.g., IRAS 18061-2505; 2 sources known, one of them without optical counterpart, and 2 candidates also obscured in the optical).

While this scenario could be valid in general, we note that geometrical effects could introduce some problems when trying to reproduce the properties of a particular object. For instance, the orientation of the object with respect to the observer affects the observational appearance of its maser emission. A source with a jet of the "water fountain" type would never exhibit its typical high-velocities if the jet was close to the plane of the sky.

Because of the limited number of objects in each of the proposed stages, it is evident that greater efforts should be made to identify obscured evolved objects harbouring water masers. The possibility of finding new "water fountains" among them (postAGB or $\mathrm{PN}$ ) or new $\mathrm{H}_{2} \mathrm{O}$-PNe seems to be higher for obscured objects and these studies would contribute to confirm or reject the proposed evolutionary scenario, and constrain the time spent by the stars in each stage.

\section{Conclusions}

We have performed a survey for water masers in a sample of 74 evolved stars selected from the SGM atlas, using the Parkes 64-m antenna.

We have detected four new water masers, three of them in obscured PNe candidates: IRAS 12405-6219, IRAS 15103-5754, and IRAS 16333-4807; and one in an obscured post-AGB candidate: IRAS 13500-6106. This could increase the number of $\mathrm{H}_{2} \mathrm{O}-\mathrm{PN}$ known from three to six.

One of the PN candidates in which water masers have been found, IRAS 15103-5754, also has the characteristics of a water fountain. If its PN nature and its association with water maser emission is confirmed, it would be the first known case of a "water fountain" $\mathrm{PN}$.

We propose an evolutionary scenario for water masers in the post-AGB and PNe stages, in which we try to accommodate all the detections in our survey. After a period in which a post-AGB star could maintain the remaining water masers produced during the AGB phase, some of them may develop water fountain characteristics. The "water fountain" masers would still be present in very young bipolar PNe. Later in the PN stage, lower-velocity water masers would exist first both along jets and close to the 
central object, with only the central masers remaining in more evolved PNe.

In this proposed scenario for the evolution of the water masers in the post-AGB and PN stages, IRAS 15103-5754 could represent the "missing link" between water fountains and $\mathrm{H}_{2} \mathrm{O}-\mathrm{PNe}$.

We confirm the tendency suggested in Paper I that the presence of water masers is favoured in obscured sources with massive envelopes.

Acknowledgements. We would like to thank our referee, Dr. Dieter Engels, for his careful and useful review. O.S., J.F.G., J.M.T., and G.A. are partially supported by Ministerio de Ciencia e Innovación, grants AYA2008-06189-C03 and AYA2005-08523-C03 (co-funded with FEDER funds). O.S. and L.F.M. are partially supported by Ministerio de Ciencia e Innovación, grant AYA2008-01934. O.S., J.F.G., L.F.M., J.M.T., and G.A. also acknowledge support from Consejería de Innovación, Ciencia y Empresa of Junta de Andalucía. This publication makes use of data products from the Two Micron All Sky Survey, which is a joint project of the University of Massachusetts and the Infrared Processing and Analysis Center/California Institute of Technology, funded by the National Aeronautics and Space Administration and the National Science Foundation.

\section{References}

Bedijn, P. J. 1987, A\&A, 186, 136

Bowers, P. F., Johnston, K. J., \& de Vegt, C. 1989, ApJ, 340, 479

de Gregorio-Monsalvo, I., Gómez, Y., Anglada, G., et al. 2004, ApJ, 601, 921

Deacon, R. M., Chapman, J. M., Green, A. J., et al. 2007, ApJ, 658, 1096

Engels, D. 2002, A\&A, 388, 252

Engels, D., Schmid-Burgk, J., \& Walmsley, C. M. 1986, A\&A, 167, 129
García-Lario, P., Manchado, A., Pych, W., et al. 1997, A\&AS, 126, 479

García-Hernández, D. A., Perea-Calderón, J. V., Bobrowsky, M., et al. 2007, ApJ, 666, L33

Gómez, J. F., Suárez, O., Gómez, Y., et al. 2008, AJ, 135, 2074

Gómez, Y., Moran, J. M., \& Rodríguez, L. F. 1990, Rev. Mex. Astron. Astrof., 20,55

Hughes, V. A., \& MacLeod, G. C. 1989, AJ, 97, 786

Imai, H. 2007, Astrophysical Masers and their Environments, ed. W. Baan, \& J.

Chapman (Cambridge: Cambridge Univ. Press), IAU Symp., 242, 279

Imai, H., Sahai, R., \& Morris, M. 2007, ApJ, 669, 424

Josselin, E., Mauron, N., Planesas, P., et al. 2000, A\&A, 362, 255

Leão, I. C., de Laverny, P., Mékarnia, D., de Medeiros, J. R., \& Vandame, B. 2006, A\&A, 455, 187

Lewis, B. M. 1989, ApJ, 338, 234

Miranda, L. F., Gómez, Y., Anglada, G., et al. 2001, Nature, 414, 284

Ratag, M. A., Pottasch, S. R., Zijlstra, A. A., et al. 1990, A\&A, 233, 181

Reid, M. J., \& Moran, J. M. 1981, ARA\&A, 19, 231

Reid, M. J., Muhleman, D. O., Moran, J. M., Johnston, K. J., \& Schwartz, P. R. 1977, ApJ, 214, 60

Sahai, R., Morris, M., Sánchez Contreras, C., et al. 2007, AJ, 134, 2200

Sevenster, M. N. 2002, AJ, 123, 2772

Suárez, O. 2004, Ph.D. Thesis, Universidad de Vigo, Spain

Suárez, O., García-Lario, P., Manchado, A., et al. 2006, A\&A, 458, 173 (Paper I)

Suárez, O., Gómez, J. F., \& Morata, O. 2007, A\&A, 467, 1085

Suárez, O., Gómez, J. F., \& Miranda, L. F. 2008, ApJ, 689, 430

Tafoya, D., Gómez, Y., Anglada, G., et al. 2007, AJ, 133, 364

Te Lintel Hekkert, P., \& Chapman, J. M. 1996, A\&AS, 119, 459

Te Lintel Hekkert, P., Caswell, J. L., Habing, H. J., Haynes, R. F., \& Norris, R. P. 1991, A\&AS, 90, 327

van de Steene, G. C. M., \& Pottasch, S. R. 1993, A\&A, 274, 895

van der Veen, W. E. C. J., Habing, H. J., \& Geballe, T. R. 1989, A\&A, 226, 108

Zijlstra, A. A., Te Lintel Hekkert, P., Pottasch, S. R., et al. 1989, A\&A, 217, 157 